\title{
37. TIPIFICACIÓN DEL NOMBRE LINNEANO PRENANTHES MURALIS L. (ASTERACEAE)
}

\author{
José Antonio MEJÍAS
}

Recibido el 15 de octubre de 2015, aceptado para su publicación el 3 de noviembre de 2015

\section{Typification of the Linnean name Prenanthes muralis L. (Asteraceae)}

Palabras clave. Cicerbita, epítipo, Lactuca, lectotipo, Linneo, Mycelis, nomenclatura, Prenanthes.

Key words. Cicerbita, epitype, Lactuca, lectotype, Linnaeus, Mycelis, nomenclature, Prenanthes

Con el nombre Prenanthes muralis, Linneo (1753) designó un taxón con categoría de especie ampliamente reconocido dentro de la familia Asteráceas, pero de clasificación difícil aún en la actualidad. Se trata de una hierba perenne que puede alcanzar más de $1 \mathrm{~m}$ de altura, con un pequeño rizoma basal que origina una roseta de hojas generalmente liradas; en primavera o verano desarrolla un tallo florífero, a veces dos o tres, terminado(s) por una panícula laxa con decenas o centenares de capítulos, todos ellos constituidos por cinco flores liguladas que originan aquenios rostrados rematados por un vilano de pelos escábridos. Se distribuye por gran parte de Europa alcanzando el Cáucaso y los Montes Urales por el este, la Península Ibérica por el oeste, Noruega por el norte y las cordilleras norteafricanas por el sur. Es, además, una especie introducida en América del Norte y Nueva Zelanda (Clabby \& Osborne, 1999).

La asignación del taxón al género Prenanthes por parte de Linneo debió estar determinada por el reducido número de flores en sus capítulos y la organización del involucro, pero a finales del siglo XVIII la combinación Prenanthes muralis dejó de ser utilizada. Habitualmente se ha considerado que esta especie está evolutivamente asociada al género Lactuca, pero para su clasificación se han utilizado criterios muy variables. Así, con frecuencia se ha aplicado un concepto amplio de Lactuca (p.ej., Stebbins 1937), combinándose el nombre bajo este género como L. muralis (L.) Gaertn., Fruct. Sem. Pl. 2: tab.185 (1795). Sin embargo, dado que sus aquenios muestran un pico perfectamente definido pero inusualmente corto (menor de 1/3 de la longitud del cuerpo), que las brácteas del involucro forman dos series muy bien diferenciadas claramente dispares y que el vilano se ha considerado dimórfico diversos autores (p.ej., Feráková 1976, Bremer 1994) han preferido considerarlo dentro de Mycelis Cass. como M. muralis (L.) Dumort., Fl. Belg. 60 (1827). Esta opción es, posiblemente, la más común, y supone la validación del género Mycelis que, en tal caso, hoy se consideraría monotípico (Bremer loc. cit.). Otros autores, los menos, han preferido combinar el nombre bajo Cicerbita Wallr. [C. muralis (L.) Wallr., Sched. Crit. 436 (1822)], un género delimitado de forma imprecisa y en el que actualmente se incluye un amplio grupo de taxones de origen eurasiático, especialmente

Este trabajo se ha desarrollado en el marco del proyecto Flora Iberica IX(2) del Plan Nacional de Investigación de 2012 del Ministerio de Economía y Competitividad CGL2012-32914, cofinanciado por Fondos FEDER. 


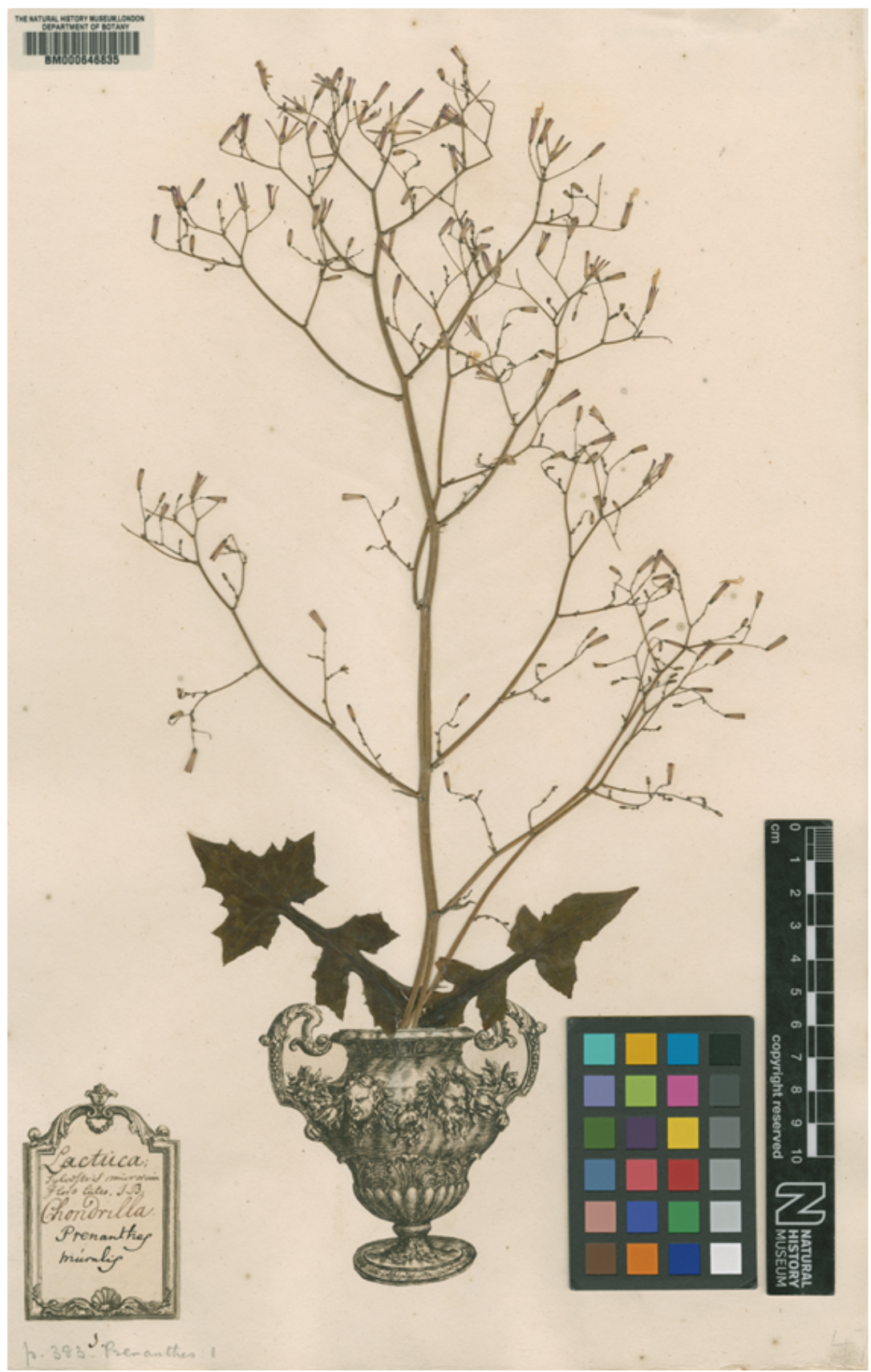

Figura 1. Lectotipo de Prenanthes muralis L., Herb. Cliff. BM00646835 (BM-Clifford). Fotografía por cortesía del Museo de Historia Natural, Londres; reproducción con permiso. Lectotype of Prenanthes muralis L., Herb. Cliff. BM00646835 (BM-Clifford). Image by courtesy of Natural History Museum, London; reproduced with permission. 
diversificado en zonas montañosas de Asia sudoccidental y central (Bremer loc.cit.).

Esta disparidad de criterios está relacionada con dificultades intrínsecas en la clasificación de la subtribu Lactucinae, a la que inequívocamente pertenece el taxón (Kilian et al. 2009). Se trata de un grupo con historia evolutiva aún poco conocida y de clasificación compleja (Kilian et al.. loc.cit., Wang et al., 2013), en el que la delimitación de los géneros no está bien resuelta, siendo un factor clave para ello la consecución de circunscripción estable para el género Lactuca. Entre las diversas razones que contribuyen a esta situación cabe citar el reducido muestreo de especies en los análisis filogenéticos, dificultado por la distribución geográfica del grupo. A pesar de ello, Koopman (2005) y Koopman et al. (1998, 2001), utilizando marcadores AFLP y secuencias nucleares ITS, han mostrado que constituye un clado bien diferenciado dentro de la subtribu, en la que ocupa una posición relativamente basal en relación a las especies más conocidas del género Lactuca. Esta posición es similar al de algunas otras especies del género Cicerbita, como C. alpina (L.) Wallr., con pico corto o mal definido.

Como se deduce de los párrafos anteriores, todos los nombres propuestos para este taxón son estrictamente sinónimos nomenclaturales, que de acuerdo con el artículo 7.3 del Código Internacional de Nomenclatura Botánica (McNeill et al. 2012), comparten el tipo nomenclatural del nombre Prenanthes muralis L., por lo que la tipificación debe realizarse sobre éste. Jarvis (2007: 769) indicó la falta de designación de un tipo para el nombre de Linneo y no tenemos noticia de que posteriormente se haya producido tal tipificación, por lo que se efectúa seguidamente.

Tipificación. El protólogo de Linneo (1753: $797)$ incluye una sucinta frase diagnóstica: "PRENANTHES flosculis quinis, foliis lyratohastata" (Prenanthes con flósculos en grupos de cinco, hojas lirado-hastadas) e indica varias obras donde es citada previamente, entre las que se encuentra Hortus Cliffortianus ... (Linneo 1738: 383). Además, indicó los sinónimos "Sonchus laevis laciniatus muralis parvis floribus", incluido en la obra Pinax theatri botanici ... de Bauhin (Bauhin 1623: 124), y "Sonchus laevis vulgatior" de la obra Rariorum plantarum historia de L'Écluse (L'Écluse 1601, 5: 146), aunque con una pequeña errata en la indicación de la página, consignada como 136 en lugar de 146.

De acuerdo con lo expuesto, Linneo pudo utilizar para la descripción de esta especie los especímenes de su herbario personal (LINN), los disponibles en el Herbario de Clifford (BM-Clifford) y los correspondientes al herbario de J. Burser (UPS-BURSER), ya que la sinonimia de la obra de Bauhin (loc.cit.) se considera referencia indirecta a este material (Jarvis, 2007). Por otro lado, en la obra de L'Écluse (loc.cit.) hay una ilustración que también puede considerarse como elemento original para la descripción. Para la tipificación, pues, disponemos en la actualidad de los siguientes materiales:

(1) Un pliego en el Herbario de la Sociedad Linneana de Londres (LINN No 952.4; http:// linnean-online.org/9677/), que muestra en su base la indicación manuscrita "4. muralis", coincidente con la numeración de Species Plantarum de 1753. De acuerdo con Turland \& Jarvis (1997) y Jarvis (2007), tal indicación permite suponer que el material fue utilizado en la descripción de la especie de la primera edición de esta obra. Además, no se ha detectado ninguna otra indicación que permita suponer que el material fue incorporado con posterioridad al herbario. El pliego incluye un fragmento bastante completo de un individuo con botones florales y capítulos en antesis pero con las lígulas de las flores mal conservadas; además, aparecen tres hojas caulinares superiores en buen estado, de las cuales una es claramente lirada y con el lóbulo terminal 


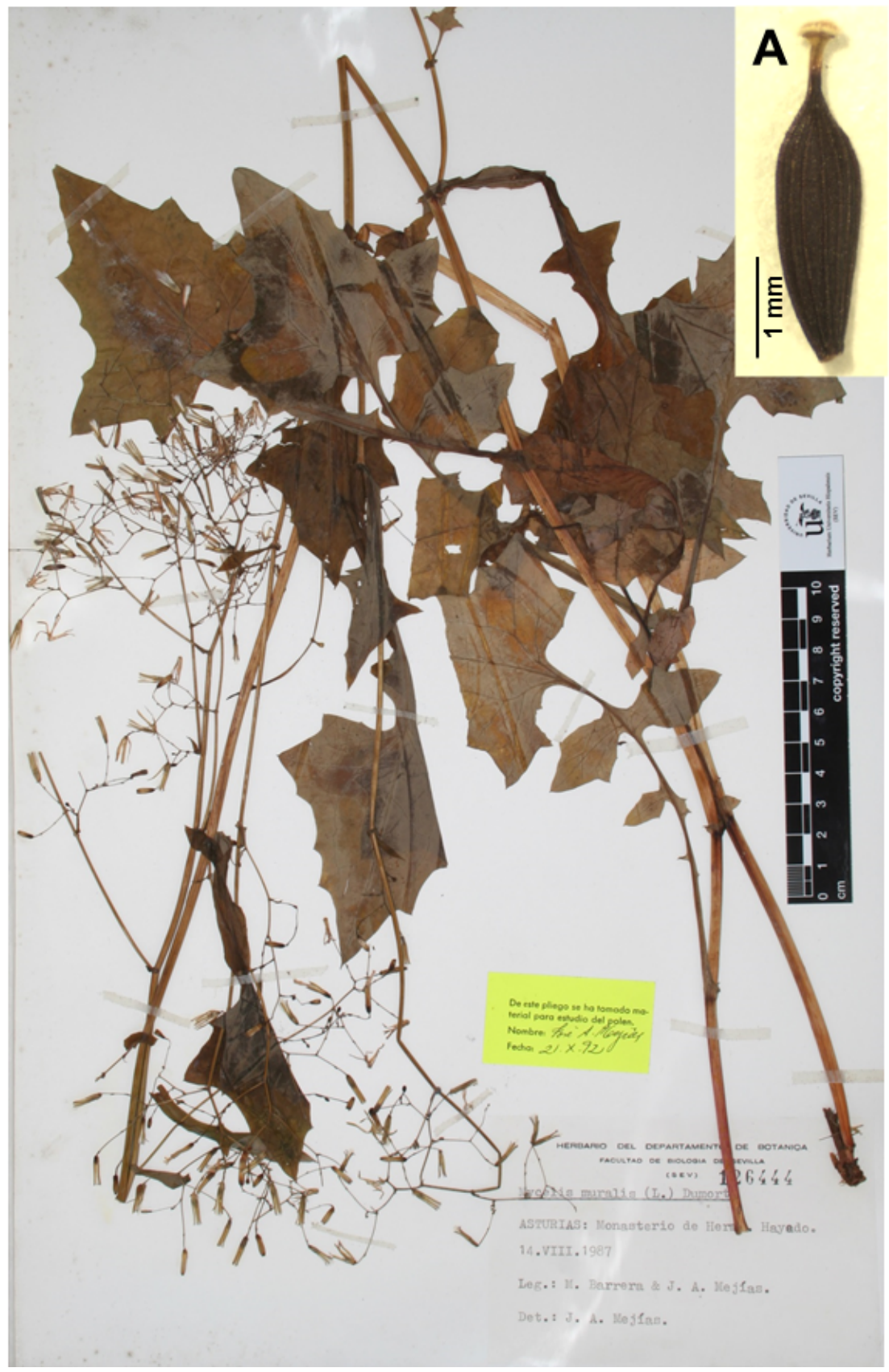

Figura 2. Epitipo de Prenanthes muralis L., Herb. Universidad de Sevilla (SEV 126444). Fotografía por cortesía del Herbario de la Universidad de Sevilla; reproducción con permiso. A) Aquenio maduro. Epitype of Prenanthes muralis L., Herb. University of Seville (SEV 126444). Image by courtesy of the Herbarium of University of Seville; reproduced with permission. A) Mature achene. 
hastado. También incluye un fragmento de tallo con dos hojas, una de las cuales es lirada pero con lóbulo terminal orbicular.

(2) Un pliego en el herbario de Clifford (BM00646835; http://www.nhm.ac.uk/ resources/research-curation/projects/cliffordherbarium/lgimages/BM000646835.JPG) que lleva en la base claramente el nombre Prenanthes muralis. Este material está constituido por una inflorescencia bastante robusta con diversos capítulos en flor bien conservados, alguno de ellos en fase de post-antesis, de manera que se pueden apreciar el número de flósculos incluidos en el capítulo. Igualmente, cuenta con dos hojas liradas cuyos lóbulos terminales son claramente hastados.

(3) Un espécimen en el herbario de Burser, UPS-BURSER 6:19 [UPS:BOT:V173205], considerado como material original por Ch. Jarvis (2007: 769), que incluye un único ejemplar con hojas liradas y lóbulos terminales más o menos hastados, además de una pequeña sinflorescencia separada que podría corresponder al mismo individuo. Ésta incluye algunos botones florales en diversas fases de desarrollo, no habiendo alcanzado ninguno de ellos la floración. El pliego incluye una etiqueta manuscrita encabezada por el nombre "Sonchus laevior lacinius parvis floribus Bauh."

(4) Una ilustración en la obra de Ch. de l'Écluse (1601, vol.5: CXLVI) de un espécimen completo con el nombre Sonchus laevior vulgaris II, en la que se muestran diversos capítulos donde se aprecia claramente la presencia de cinco lígulas y varias hojas liradas con lóbulo terminal hastado, aunque el porte resulta más robusto del natural de la especie, con tallos inusualmente gruesos.

De los especímenes conservados en pliego, el del Herbario Clifford (BM00646835) es el que muestra con mayor claridad los caracteres diagnósticos propuestos por Linneo, y está bien conservado, por lo que se considera el mejor material original que se pudo utilizar para la descripción de Prenanthes muralis, y se escoge aquí como lectotipo, elección que se aprecia más idónea que hacerlo de la ilustración de L'Écluse (1601). Sin embargo, ninguno de los materiales de herbario a que se ha hecho referencia posee fruto y, de manera similar, en el icono de l'Écluse (loc.cit.) no se representa aquenio alguno. Puesto que los frutos son de gran importancia para el reconocimiento y asignación de los géneros y las especies dentro de la subtribu Lactucinae, se ha designado aquí un epitipo acorde con el Art. 9.8 del Código Internacional de Nomenclatura Botánica (McNeill et al. 2012). Se trata de un espécimen conservado en el herbario de la Universidad de Sevilla (SEV 126444), que presenta dos tallos no ramificados y sendas inflorescencias terminales, recolectado a finales del siglo pasado en fase de fructificación y en el que, además, se pueden comprobar los caracteres propuestos por Linneo. Este espécimen fue utilizado en un estudio de morfología polínica del género Lactuca y taxones relacionados en la Península Ibérica (Moreno-Socías et al. 1994) y a partir de sus semillas se obtuvieron plantas en las se analizó el número cromosómico de las especie, la morfología de sus cromosomas y las características del cariotipo (Mejías 1993).

Prenanthes muralis L., Sp. Pl.: 797 (1753)

Ind. loc.: "Habitat in Europe nemoribus umbrosis"

Lectotypus (hic designatus): Herb. Cliff. BM00646835 (BM-Clifford) (fig. 1) (imagen disponible en www.nhm.ac.uk/resources/ research-curation/projects/clifford-herbarium/ lgimages/BM000646835.JPG).

Epitypus (hic designatus): Spain, Asturias: Monasterio de Hermo. Hayedo. 14.VIII.1987. M. Barrera \& J.A. Mejías (SEV barcode SEV 126444, fig. 2).

AGRADECIMIENTOS. Quisiera manifestar 
mi sincero agradecimiento al Prof. Salvador Talavera (Universidad de Sevilla), que me impulsó a desarrollar este trabajo y me proporcionó la información necesaria para plantearlo; igualmente, agradezco los consejos del Dr. C. Romero Zarco y la revisión del Prof. J.A. Devesa. Ha sido de gran ayuda la colaboración y disponibilidad del Dr. Mats Hjertson (Museum of Evolution, Botany Section Uppsala University, Suecia), que me facilitó la posibilidad de estudiar los materiales incluidos en el Herbario de Burser (UPS).

\section{BIBLIOGRAFÍA}

BREMER, K. -1994- Asteraceae: Cladistics and Classification. Timber Press, Portland, Oregon.

CLABBY, G. \& B. OSBORNE -1999- Biological flora of the British Isles: Mycelis muralis (L.) Dumort. (Lactuca muralis (L.) Gaertner. J. Ecol, 87: 156-172.

FERÁKOVÁ, V. -1976- Genus Mycelis in Th. Tutin et al. (eds.), Flora Europaea 5: 331-332.

JARVIS, C. -2007- Order out of chaos: Linnean plant names and their types. Linean Society of London and the Natural History Museum, London.

KILIAN, N., B. GEMEINHOLZER \& H.W. LACK -2009- V.A. Funk, A. Susanna, T.F. Stuessy \& R.J. Bayer (eds.): Systematics, evolution and biogeography of Compositae: 343-383. International Association for Plant Taxonomy, Viena.

KOOPMAN, W.J.M. -2005- Phylogenetic signal in AFLP data sets. Syst. Biol. 54: 197-217.

KOOPMAN, W.J.M., E. GUETTA, C.C.M. VAN DE WIEL, B. VOSMAN \& R.G. VAN DER BERG -1998- Phylogenetic relationships among Lactuca (Asteraceae) species and related genera based om ITS-1 DNA sequences. Amer. J. Bot. 85: 1517-1530.

KOOPMAN, W.J.M., M.J. ZEBENBERGEN \& R.G. VAN DER BERG -2001- Species realtionships in Lactuca s.1. (Lactuceae, Asteraceae) inferred from AFLP fingerprints. Amer. J. Bot. 88: 1881-1887.

L'ÉCLUSE, CH. DE -1601- Rariorum plantarum historia, 5 vols. Amberes.

LINNEO, C. -1738- Hortus Cliffortianus. Amsterdam.

LINNEO, C. -1753- Species Plantarum, 2 vols. Stockholm.
MCNEILL, J., F.R. BARRIE, W.R. BUCK, V. DEMOULIN, W. GREUTER, D.L. HAWKSWORTH, P.S. HERENDEEN, S. KNAPP, K. MARHOLD, J. PRADO, W.F. PRUD'HOMME VAN REINE, G.F. SMITH, J.H. WIERSEMA \& N.J. TURLAND (eds.) -2012- International Code of nomenclature for algae, fungi, and plants (Melbourne Code). Regnum Vegetabile 154. Koeltz Scientific Books (www.iapt-taxon.org/nomen/main.php).

MEJÍAS, J.A. -1993- Mediterranean chromosome number reports: Prenanthes, Cicerbita and Mycelis in the Iberian Peninsula. Fl. Medit. 3: 333-338.

MORENO-SOCÍAS, E., J.A. MEJÍAS \& M.J. DÍEZ -1994- Morfología polínica de Lactuceae (Asteraceae) en la Península Ibérica. I. Lactuca L. y géneros relacionados. Acta Bot. Malac. 19: 103-113.

STEBBINS, G.L. -1937- Critical Notes on Lactuca and related genera. J. Bot.: 75: 12-18.

TURLAND, N.J. \& C.E. JARVIS -1997Typification of Linnean specific and varietal names in the Leguminosae (Fabaceae). Taxon 46: 457-485.

WANG, Z.H., H. PENG \& N. KILIAN -2013Molecular phylogeny of the Lactuca Alliance (Cichorieae Subtribe Lactucinae, Asteraceae) with focus on their chinese centre of diversity detects potential events of reticulation and chloroplast capture. Plos One 8: e82692.

Dirección del autor: Departamento de Biología Vegetal y Ecología, Universidad de Sevilla. Avda. Reina Mercedes, 6. Apartado 1095, 41080 Sevilla (Spain).jmejias@us.es 